\title{
Quasiparticle Trapping in Microwave Kinetic Inductance Strip Detectors
}

\author{
D. C. Moore*, B. A. Mazin ${ }^{\dagger}$, S. Golwala*, B. Bumble**, J. Gao*, B. A. Young ${ }^{\S}$, \\ S. McHugh ${ }^{\dagger}$, P. K. Day ${ }^{* *}$, H. G. LeDuc ${ }^{* *}$ and J. Zmuidzinas* \\ *Physics Department, California Institute of Technology, Pasadena, CA 91125 \\ ${ }^{\dagger}$ Department of Physics, University of California, Santa Barbara, CA 93106 \\ ** Jet Propulsion Laboratory, California Institute of Technology, Pasadena, CA 91109 \\ ${ }^{\ddagger}$ National Institute of Standards and Technology, Boulder, CO 80305

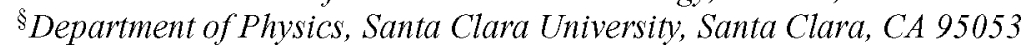

\begin{abstract}
Microwave Kinetic Inductance Detectors (MKIDs) are thin-film, superconducting resonators, which are attractive for making large detector arrays due to their natural frequency domain multiplexing at GHz frequencies. For X-ray to IR wavelengths, MKIDs can provide high-resolution energy and timing information for each incoming photon. By fabricating strip detectors consisting of a rectangular absorber coupled to MKIDs at each end, high quantum efficiency and spatial resolution can be obtained. A similar geometry is being pursued for phonon sensing in a WIMP dark matter detector. Various materials have been tested including tantalum, tin, and aluminum for the absorbing strip, and aluminum, titanium, and aluminum manganese for the MKID. Initial Ta/Al X-ray devices have shown energy resolutions as good as $62 \mathrm{eV}$ at 6 $\mathrm{keV}$. A Ta/Al UV strip detector with an energy resolution of $0.8 \mathrm{eV}$ at $4.9 \mathrm{eV}$ has been demonstrated, but we find the coupling of the MKIDs to the absorbers is unreliable for these thinner devices. We report on progress probing the thicknesses at which the absorber/MKID coupling begins to degrade by using a resonator to inject quasiparticles directly into the absorber. In order to eliminate the absorber/MKID interface, a modified design for implanted AlMn/A1 UV strip detectors was developed, and results showing good transmission of quasiparticles from the absorber to MKID in these devices are presented.
\end{abstract}

Keywords: Microwave kinetic inductance detectors, strip detectors, WIMP dark matter detectors, quasiparticle trapping PACS: $85.25 . \mathrm{Oj}, 95.35 .+\mathrm{d}$

\section{INTRODUCTION}

Microwave kinetic inductance detectors (MKIDs) are high-Q, superconducting resonators which respond to changes in the quasiparticle density in the superconductor through a change in surface impedance. We have demonstrated the detection of submillimeter, optical/UV, and X-ray photons using MKIDs [1-3]. For X-ray and optical/UV astronomy, we are pursuing spectrophotometer arrays based on a strip detector architecture $[4,5]$. In a strip detector, photons are absorbed in a rectangular strip creating quasiparticles, which diffuse to either end of the strip where they are sensed by MKIDs. If the MKIDs are made of a lower gap material than the $a b-$ sorbing strip, the quasiparticles quickly emit a phonon and fall below the gap of the absorber, preventing diffusion out of the MKID. Since the response of the detector is proportional to the density of quasiparticles in the MKID, quasiparticle trapping allows for sensitive detectors while still maintaining large volume absorbers with high absorption efficiency.

Quasiparticle trapping is also used by the Cryogenic Dark Matter Search (CDMS) ZIP detectors for sensing athermal phonons produced by the interaction of Weakly Interacting Massive Particles (WIMPs) [6, 7]. The strip detector design described above can be adapted to a CDMS style phonon detector by running MKIDs along the edges of $\sim 1 \mathrm{~mm}$ wide $\mathrm{Al}$ absorbers [8]. An MKIDbased phonon detector would allow for finer pixellation of the phonon sensors as well as double-sided detector coverage due to their natural frequency domain multiplexing. Additionally, the cryogenic complexity of the readout electronics would be significantly reduced since the multiplexing would be performed by warm, largely commercial hardware $[9,10]$.

\section{STRIP DETECTORS}

The geometry of the X-ray and optical/UV strip detectors is shown in Fig. 1a. The rectangular absorbing strip (typically made of $\mathrm{Ta}$ ) is $30 \mu \mathrm{m}$ wide and several hundred $\mu \mathrm{m}$ long. MKIDs (typically made of Al, AlMn, or Ti) are attached to both ends of the strip, with a $15 \mu \mathrm{m}$ long triangular trapping region made of the same material as the MKID. When a photon is absorbed in the Ta strip, quasiparticles diffuse and are trapped in the MKIDs, changing the surface impedance of the resonator and causing a shift in the amplitude and phase of the microwave signal transmitted past the resonator. The sum of the responses

\footnotetext{
CP1185, Low Temperature Detectors LTD 13, Proceedings of the $13^{\text {th }}$ International Workshop

edited by B. Cabrera, A. Miller, and B. Young

C 2009 American Institute of Physics 978-0-7354-0751-0/09/\$25.00
} 


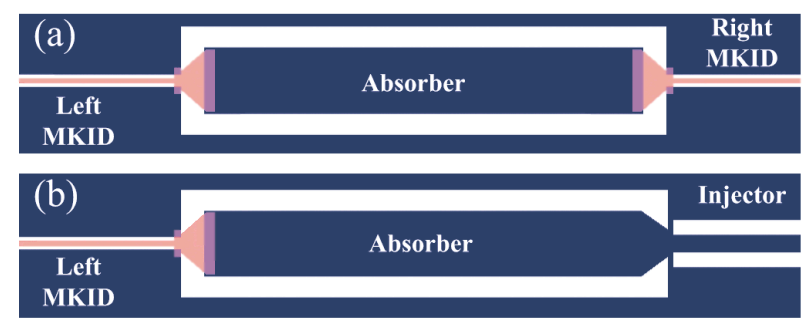

FIGURE 1. (Color online) (a) Geometry of the strip detector devices. The absorbing strip, shown in blue, is $30 \mu \mathrm{m}$ wide, and several hundred $\mu \mathrm{m}$ long. The MKIDs, shown in red, are attached to each end of the absorber. (b) Geometry of the quasiparticle injectors. For the injector device, the right MKID in the strip detector design is replaced with a resonator made of the same material as the absorbing strip.

in both MKIDs is proportional to the total photon energy, and the ratio of the responses gives the location in the strip where the photon was absorbed.

We have demonstrated working strip detectors in both the X-ray and optical/UV. The fabrication procedure for $\mathrm{X}$-ray and optical devices differs only in the thicknesses of the films deposited, and is described in detail in Ref. [3]. Before the deposition of the $\mathrm{Al} \mathrm{film}$, the Ta is argon ion milled in situ at $150 \mathrm{eV}$ with a current density of $0.25 \mathrm{~mA} / \mathrm{cm}^{2}$ for $1-3$ minutes to remove any native oxide.

Fig. 2a shows the pulse heights in the left and right MKIDs for a Ta/Al strip detector illuminated by an ${ }^{55} \mathrm{Fe}$ $\mathrm{X}$-ray source. The detector consisted of a $200 \mu \mathrm{m}$ long, $600 \mathrm{~nm}$ thick Ta absorber coupled to $200 \mathrm{~nm}$ thick Al MKIDs. The $\mathrm{Mn} K_{\alpha}$ and $K_{\beta}$ lines are both visible, with an energy resolution of $62 \mathrm{eV}$ at $6 \mathrm{keV}$, limited by noise from two-level systems (TLS) in amorphous dielectric layers on the resonator surfaces [11]. We have demonstrated that this TLS noise can be reduced by scaling the capacitive section of the resonator to larger geometries [12], and are developing fabrication techniques to reduce the number of TLS present on the resonator surfaces. From an analysis of events at varying locations along the strips, we infer a Ta diffusion constant of $13.5 \pm 1.8 \mathrm{~cm}^{2} / \mathrm{s}$ and a quasiparticle lifetime in the $\mathrm{Ta}$ of $34.5 \pm 5.7 \mu \mathrm{s}$, leading to a diffusion length in the $\mathrm{Ta}$ of $l=\sqrt{D \tau}=216 \pm 30 \mu \mathrm{m} \mathrm{[3]}$.

Fig. 2b shows the pulse heights measured for a optical/UV strip detector with $100 \mu \mathrm{m}$ long, $60 \mathrm{~nm}$ thick Ta absorbers and $20 \mathrm{~nm}$ thick Al MKIDs, illuminated by $254 \mathrm{~nm}$ UV photons. The responsivity for this device has been increased by a factor of $\sim 1000$ relative to the $\mathrm{X}$-ray detector described above. By reducing the MKID thickness from $200 \mathrm{~nm}$ to $20 \mathrm{~nm}$, and the width from 3 $\mu \mathrm{m}$ to $1 \mu \mathrm{m}$, the volume was reduced by a factor of 30 and the kinetic inductance fraction was increased from 0.03 to 0.75 , giving the necessary increase in responsiv- ity. The energy resolution for this device was $0.8 \mathrm{eV}$ at $4.9 \mathrm{eV}$. The diffusion length in the $60 \mathrm{~nm}$ thick Ta was $\sim 60 \mu \mathrm{m}$, significantly shorter than for the $600 \mathrm{~nm}$ films above, leading to losses even in the shorter $100 \mu \mathrm{m}$ strip.

We can reliably fabricate X-ray strip detectors (MKID thicknesses $\gtrsim 200 \mathrm{~nm}$ ) which show the expected correlation between pulse heights in the right and left MKIDs, indicating good transmission and trapping of quasiparticles from the absorber into the MKID. This trapping has been seen for both Ta and Sn absorbers with thicknesses between $150 \mathrm{~nm}$ and $650 \mathrm{~nm}$. However, when the same geometry and fabrication procedures are used for thin $(\sim 20 \mathrm{~nm})$ MKIDs, each MKID always shows large optical pulses, but the correlation between pulse heights in the left and right channel is not seen repeatably. For nearly all of the optical/UV devices tested, either the quasiparticles were not being transmitted through the $\mathrm{Al} / \mathrm{Ta}$ interface, or they were diffusing out of the $\mathrm{Al}$ before they could become trapped. In the following section we present evidence that the quasiparticles are not being transmitted from the Ta absorbers into the Al MKIDs.

\section{QUASIPARTICLE INJECTORS}

In order to study the quasiparticle transmission and trapping at thicknesses between the X-ray and optical/UV devices, the strip detector geometry was modified to allow the direct creation of quasiparticles by microwave power at one end of the strip. These "quasiparticle injectors" consist of a resonator made from the same material as the absorber shorted to the right side of the strip, as shown in Fig. 1b. When the injector is driven at its resonant frequency (or one of its harmonics), power is dissipated in the injector, creating quasiparticles above the gap of the absorber. These quasiparticles can then diffuse across the strip and be trapped in a lower gap MKID attached to the left side of the strip.

There are two regimes for injecting quasiparticles into the strip. When the length of the microwave pulse is much longer than the quasiparticle lifetime and diffusion time, a steady state is reached where the quasiparticle creation rate, $\Gamma_{c}=\eta_{i n j} P^{S S} / \Delta_{a b s}$, equals the decay rate, $\Gamma_{d}=N_{a b s} / \tau_{a b s}+N_{M K I D} / \tau_{M K I D}$, where $\eta_{i n j}$ is the efficiency for the microwave power to create quasiparticles, $P^{S S}$ is the steady state microwave power, $\Delta_{a b s}$ is the energy gap in the absorber, and $N_{a b s / M K I D}$ and $\tau_{a b s / M K I D}$ are the steady state number of quasiparticles and lifetime in the absorber/MKID. In this regime, a response will be seen in the MKID as long as there is quasiparticle transmission from the absorber to the MKID, even if there is no trapping, since enough quasiparticles will be created to fill the strip and MKID with a uniform density.

For pulses which are much shorter than the diffusion time, the quasiparticle density does not reach a steady 
(a)

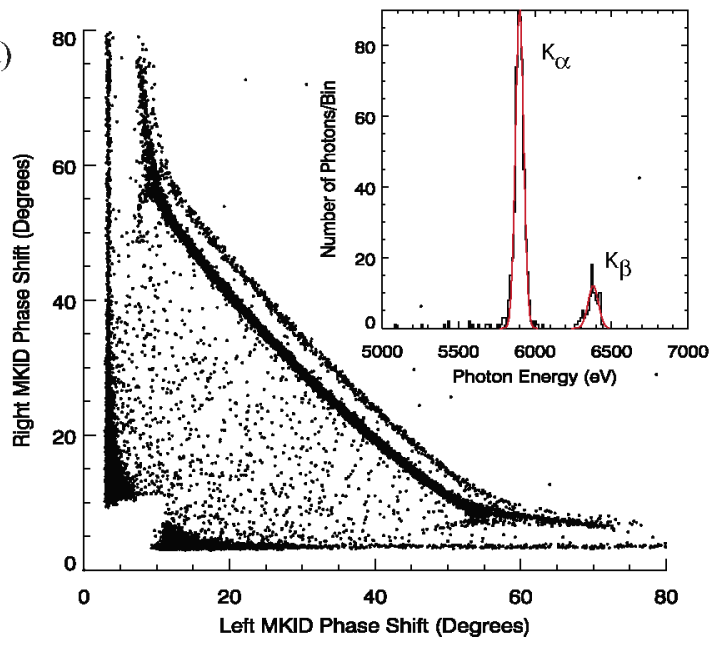

(b)

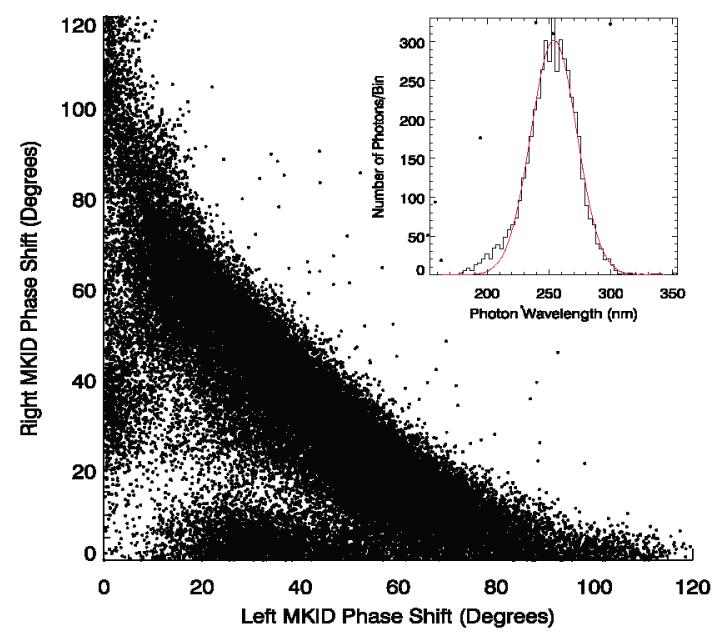

FIGURE 2. (a) Optimally filtered pulse heights for $200 \mathrm{~nm}$ thick A1 MKIDs attached to a $200 \mu \mathrm{m}$ long Ta strip, illuminated by an ${ }^{55} \mathrm{Fe} \mathrm{X}$-ray source. This figure is reproduced from Ref. [3]. (b) Optimally filtered pulse heights for $20 \mathrm{~nm}$ thick Al MKIDs attached to a $100 \mu \mathrm{m}$ long Ta strip, illuminated by $254 \mathrm{~nm}$ UV photons. The inset of each plot shows the energy spectrum for events with greater than 20 degrees of phase shift in both MKIDs.

state, and the amount of signal seen in the MKID depends strongly on the trapping efficiency. For a short pulse, the number of quasiparticles created is $N^{\text {pulse }}=$ $\eta_{\text {inj }} P^{\text {pulse }} T^{\text {pulse }} / \Delta_{a b s}$, where $P^{\text {pulse }}$ and $T^{\text {pulse }}$ are the microwave power and duration of the pulse. The number of quasiparticles in the MKID is $N_{\text {MKID }}^{\text {pulse }}=\eta_{\text {trap }} N^{\text {pulse }}$, where $\eta_{\text {trap }}$ is the quasiparticle trapping efficiency.

Initial results from $\mathrm{Al} / \mathrm{Ta}$ injector devices indicate that quasiparticles are not being transmitted from the Ta to the Al for the optical/UV devices. When driving the injectors in the steady state regime described above, a quasiparticle response was seen for X-ray devices with $320 \mathrm{~nm}$ thick Al and $160 \mathrm{~nm}$ thick Ta. However, for optical/UV devices with $25 \mathrm{~nm}$ thick $\mathrm{Al}$ and $70 \mathrm{~nm}$ thick Ta, no quasiparticle response was seen when driving the injector. Devices at intermediate thicknesses will be studied to determine the diffusion length in the Ta and trapping probability as a function of thickness.

Since the $\mathrm{Al} / \mathrm{Ta}$ interface appears to prevent quasiparticles from reaching the MKID, optical/UV strip detectors which eliminate the absorber/MKID interface were developed using implanted AlMn/Al. These devices consisted of a single layer, $60 \mathrm{~nm}$ thick $\mathrm{Al}$ film patterned into the standard strip detector geometry. The triangular trapping region and last $200 \mu \mathrm{m}$ of the MKID was then selectively implanted with $4.8 \times 10^{14} \mathrm{Mn}$ ions $/ \mathrm{cm}^{2}$ at $20 \mathrm{keV}$ using a photoresist implant mask, lowering the superconducting transition temperature of the implanted region to $500 \mathrm{mK}$ [13]. It was found that the implant also lowered the internal $Q$ s of the resonators to $\sim 2 \times 10^{4}$, but that annealing the devices in vacuum for 60 minutes at $300 \mathrm{C}$ after the implant increased the internal $Q$ s to $\sim 4 \times 10^{5}$. In addition, the $T_{c}$ was increased to $720 \mathrm{mK}$ after annealing, consistent with results from cosputtered AlMn devices [14], where the Mn concentration is constant with depth in the film.

Fig. 3 shows the pulse response in the left MKID when driving the injectors for the AlMn/Al device, demonstrating transmission of quasiparticles from the absorber to MKID in these devices. From fits to the pulse response, we find the diffusion constant, $D_{A l}=19.8 \pm 5.3 \mathrm{~cm}^{2} / \mathrm{s}$, and lifetime, $\tau_{A l M n}=130.1 \pm 4.4 \mu \mathrm{s}$. Although Barends et al. report a decrease in the lifetime in Al films implanted with $\sim 10$ times lower Mn concentrations [15], the measured lifetime in our implanted AlMn films is consistent with lifetimes previously measured for $60 \mathrm{~nm}$ Al films, so we assume $\tau_{A l} \approx \tau_{A l M n}$ and calculate a diffusion length in the $\mathrm{Al}, l=507.5 \pm 68.5 \mu \mathrm{m}$.

From the ratio of the steady state response to the pulse response, we can estimate the trapping efficiency, $\eta_{\text {trap }}$. In the limit of good diffusion and poor trapping, the steady state density of quasiparticles is constant throughout the absorber and MKID, so the quasiparticle decay rate becomes $\Gamma_{d}=N_{a b s} / \tau_{a b s}+N_{M K I D} / \tau_{M K I D}=$ $\left(N_{M K I D} / \tau_{M K I D}\right)\left(1+V_{a b s} / V_{M K I D}\right)$, where we have assumed $\tau_{M K I D} \approx \tau_{a b s} \approx 100 \mu \mathrm{s}$ as above. The ratio of volumes for the absorber and MKID for this device is $V_{a b s} / V_{M K I D}=9.6$. Since both the steady state and pulse response depend on quasiparticle creation efficiency, we can take the ratio of the steady state and pulse response to eliminate $\eta_{i n j}$ and obtain the trapping efficiency:

$$
\eta_{\text {trap }}=\left(1+\frac{V_{a b s}}{V_{M K I D}}\right)^{-1} \frac{P^{S S} \tau_{M K I D}}{P^{\text {pulse }} T^{\text {pulse }}} \frac{\delta \theta^{\text {pulse }}}{\delta \theta^{S S}}
$$

where the phase response $\delta \theta$ is proportional to the number of quasiparticles in the MKID, $N_{M K I D}$. For the 200 


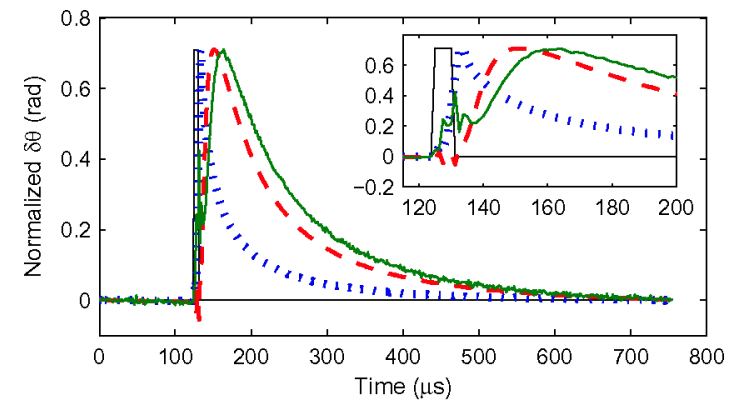

FIGURE 3. (Color online) Phase response of the left MKID for several different strip lengths when the corresponding injector was driven at its $3 \mathrm{rd}$ harmonic at a feedline power of -61 $\mathrm{dBm}$. The inset shows an enlarged version of the rising edges of the pulses. The microwave pulse is a $5 \mu$ s long square wave, shown in black. When the left MKID is driven, there is a fast pulse (blue, dotted) which peaks within a ring-up time after the microwave power is turned off. When the injector at the opposite side of the strip is driven, a slow rise time is seen. The response for the MKID attached to a $200 \mu \mathrm{m}$ strip (red, dashed) peaks $\sim 15 \mu$ s earlier than for the $400 \mu \mathrm{m}$ strip (green, solid), due to the longer diffusion time in the $400 \mu \mathrm{m}$ strip. All pulses are normalized to the pulse height for the $200 \mu \mathrm{m}$ strip. The transient oscillations visible immediately after the microwave power is switched off are due to the readout electronics and not the response of the resonators.

$\mu \mathrm{m}$ strip, the steady state phase response was $\delta \theta^{S S}=$ $0.15 \mathrm{rad}$ at a microwave drive power of $-81 \mathrm{dBm}$, and the pulse response was $\delta \theta^{\text {pulse }}=0.09 \mathrm{rad}$ for a $5 \mu$ s pulse at $-69 \mathrm{dBm}$. From this response, we determine the trapping efficiency, $\eta_{\text {trap }}=0.07$. When illuminating this device with $254 \mathrm{~nm} \mathrm{UV}$ photons, we measured 60 degree pulses for events directly absorbed in the AlMn section. The rms phase noise was measured to be $\sim 8$ degrees. By enlarging the trapping region, implanting to a higher dose to lower the AlMn $T_{C}$ to $500 \mathrm{mK}$, and using sapphire substrates with lower TLS noise, we should easily be able to observe correlated pulses for strip events for these devices.

\section{CONCLUSIONS}

Due to their inherent frequency domain multiplexing, optical/UV and X-ray MKID strip detectors are natural candidates for fabricating large detector arrays. We can reliably fabricate X-ray strip detectors with energy resolutions $\sim 60 \mathrm{eV}$, with substantial improvement possible through the reduction of two-level systems coupled to the resonator. In thinner devices sensitive to optical/UV photons, the transmission of quasiparticles from the Ta absorber to MKID is unreliable. We are investigating the thicknesses at which this transmission begins to break down by using resonators to inject quasiparticles directly into the absorber. Single layer implanted AlMn devices have shown good transmission of quasiparticles at optical thicknesses, and reliable AlMn/Al optical strip detectors should be achievable with only minor modifications to the $\mathrm{Mn}$ concentration and trapping geometry of the devices tested to date.

\section{ACKNOWLEDGMENTS}

This research was carried out at the Jet Propulsion Laboratory, California Institute of Technology, under a contract with the National Aeronautics and Space Administration. We would like to thank the JPL Research and Technology Development program, and the Gordon and Betty Moore foundation, for their generous support.

\section{REFERENCES}

1. P. Day, H. Leduc, B. Mazin, A. Vayonakis, and J. Zmuidzinas, Nature 425, 817-821 (2003).

2. J. Schlaerth, et al., Journal of Low Temperature Physics 151, 684-689 (2008).

3. B. Mazin, B. Bumble, P. Day, M. Eckart, S. Golwala, J. Zmuidzinas, and F. Harrison, Appl. Phys. Lett. 89, 222507 (2006).

4. H. Kraus, F. von Feilitzsch, J. Jochum, R. Mössbauer, T. Peterreins, and F. Pröbst, Physics Letters B 231, 195-202 (1989).

5. J. Jochum, H. Kraus, M. Gutsche, B. Kemmather, F. von Feilitzsch, and R. L. Mössbauer, Annalen der Physik 505, 611-634 (1993).

6. Z. Ahmed, et al., Physical Review Letters 102, 011301 (2009).

7. R. M. Clarke, et al., Applied Physics Letters 76, 2958 (2000).

8. S. Golwala, J. Gao, D. Moore, B. Mazin, M. Eckart, B. Bumble, P. Day, H. G. Leduc, and J. Zmuidzinas, Journal of Low Temperature Physics 151, 550-556 (2008).

9. B. Mazin, P. Day, K. Irwin, C. Reintsema, and J. Zmuidzinas, AIP Conference Proceedings 559, 799-801 (2006).

10. S. J. C. Yates, A. M. Baryshev, J. J. A. Baselmans, B. Klein, and R. Güsten, Applied Physics Letters 95, 042504 (2009).

11. J. Gao, J. Zmuidzinas, B. Mazin, H. LeDuc, and P. Day, Appl. Phys. Lett. 90, 102507 (2007).

12. O. Noroozian, these proceedings (2009).

13. B. A. Young, J. R. Williams, S. W. Deiker, S. T. Ruggiero, and B. Cabrera, Nuclear Instruments and Methods in Physics Research A 520, 307-310 (2004).

14. S. W. Deiker, et al., Applied Physics Letters 85, 2137 (2004).

15. R. Barends, S. van Vliet, J. J. A. Baselmans, S. J. C. Yates, J. R. Gao, and T. M. Klapwijk, Phys. Rev. B. 79, 020509 (2009). 